\title{
Formación y desarrollo de competencias gerenciales para los gestores hoteleros*
}

\author{
Management training and skills development for hotel managers \\ Formation et développement des compétences en gestion pour \\ les directeurs hôteliers
}

Luis A. Castro Milano

castromilano@gmail.com

Universidad de Oriente,

Isla de Margarita - Venezuela

Doctor en Administración, Universidad Del Sur, Estados

Unidos Mexicanos, 2012.

Profesor Agregado Adscrito al Departamento de Servicios

Turísticos de la Escuela de

Hotelería y Turismo, Núcleo de Nueva Esparta, Universidad

de Oriente, Venezuela.

Investigador adscrito al Centro de Investigaciones Turísticas

(CITUR). Investigador "A" acreditado por el Programa de Estímulo a la Innovación

e Investigación (PEII) del

Ministerio del Poder Popular

para Ciencia, Tecnología

e Industrias Intermedias,

Venezuela. Coordinador de los

Cursos Especiales de Grado de

la Licenciatura en Hotelería.

Coordinador del Área de

Hospitalidad de la Licenciatura en Hotelería.

Artículo de investigación científica y tecnológica

Según Clasificación Colciencias

Fecha de recepción: $14 / 08 / 2012$

Fecha de corrección: $16 / 11 / 2012$

Fecha de aprobación: $10 / 12 / 2012$

\section{Resumen}

El talento humano es la piedra angular para propiciar desde las "nuevas lógicas" empresariales respuestas a las necesidades de cambios en el comportamiento de la relación binomial oferta-demanda en los hoteles dedicados a la gestión y producción de servicios puros basados en las personas. Por esta razón se necesitan gestores concienzudos, éticos, motivados, capacitados y comprometidos con la satisfacción de los clientes internos y externos. El presente artículo de investigación científica y tecnológica, fundamentado en el eje temático gestión del talento humano y liderazgo, profundiza en la formación y el desarrollo de las competencias gerenciales del talento humano en puestos de trabajo de gestores, en los establecimientos hoteleros inscritos en el Registro Turístico Nacional (RTN) y categorizados como hoteles cinco estrellas. El discurso está fundamentado en una investigación aplicada de campo y transeccional, realizada en Venezuela, en el estado Nueva Esparta, específicamente en la Isla de Margarita. La disertación concluye demostrando la necesidad de emprender de forma inmediata la formación y el desarrollo de las competencias gerenciales en los establecimientos de alojamiento turístico. Se recomienda aprovechar los incentivos tributarios establecidos en la legislación venezolana para financiar las actividades de formación y desarrollo de las competencias gerenciales.

Palabras clave: competencias, desarrollo, formación, gestores, hoteles, turismo.

\footnotetext{
* El presente artículo es parte del resultado de una investigación realizada por cinco años, para la tesis doctoral, aprobada con alto rendimiento académico en la Universidad Del Sur, Estados Unidos Mexicanos, 2012.
} 


\section{Management training and skills development for hotel managers}

Formación y desarrollo de competencias gerenciales para los gestores hoteleros

Formation et développement des compétences en gestion pour les directeurs hôteliers

\section{Abstract}

Human talent is the cornerstone to promote responses from the entrepreneurial "new logic" to the needs of changes in the behavior of the binomial relationship between supply and demand in hotels dedicated to the management and service delivery based on people. For this reason, conscientious, ethical, motivated, and trained managers are needed committed to satisfying internal and external customers. This scientific and technological research article, based on the theme: human resource management and leadership, explores the formation and development of managerial competencies in human talent management jobs in the hotels listed in the National Tourism Registry (RTN, for the term in Spanish) and categorized as five star hotels. The discourse is based on applied field and transactional research, conducted in Venezuela in the state of Nueva Esparta, specifically in Margarita Island. The dissertation concludes by demonstrating the need to immediately undertake the formation and development of managerial skills in tourist accommodation establishments. It recommends taking advantage of tax incentives established by Venezuelan legislation to fund training and development of managerial competencies.

Keywords: development, hotels, managers, skills, tourism, training.
Formation et développement des compétences en gestion pour les directeurs hôteliers

Formación y desarrollo de competencias gerenciales para los gestores hoteleros

Management training and skills

development for hotel managers

\section{Résumée}

Le talent humain est la pierre angulaire pour rendre propice, à partir des "nouvelles logiques" entrepreneuriales, des réponses aux besoins de changement du comportement de la relation binomiale offre-demande dans les hôtels dédiés à la gestion et à la production de services sur la base des personnes. C'est pour cela qu'on a besoin de directeurs consciencieux, éthiques, motivés, capacités et compromis avec la satisfaction des clients internes et externes. Cet article de recherche scientifique et technologique, basé sur l'axe thématique de la gestion du talent humain et du leadership, approfondit la formation et le développement des compétences de gestion du talent humain sur des postes de travail des directeurs, dans les établissements hôteliers inscrits sur le Registre Touristique National (RTN) et catégorisés comme hôtels à cinq étoiles. Le discours repose sur une recherche transactionnelle appliquée sur le terrain, réalisée au Venezuela, dans l'état de Nueva Esparta, spécifiquement dans l'île Margarita. La dissertation finit par démontrer le besoin de commencer immédiatement la formation et le développement de compétences de gestion dans les établissements de tourisme. Il est recommandable de profiter les incitations tributaires établies sur la législation vénézuélienne pour financer les activités de formation et de développent des compétences en gestion.

Mots clef: compétences, développement, formation, directeurs, hôtels, tourisme. 


\section{Formación y desarrollo de competencias gerenciales para los gestores hoteleros}

\section{Introducción}

El exacerbado nivel de competitividad actual, impulsado por los procesos estructurales de la globalización, creciente desregularización, apertura de los mercados, rápida maduración del comercio electrónico y el auge de las telecomunicaciones, ha dado mayor poder a los consumidores para estar informados e impacientes por conseguir la satisfacción de sus necesidades, deseos, expectativas e incluso sus fantasías. Desde esta perspectiva, cada día aumentan los niveles de exigencias de los clientes haciendo que las empresas deban responder proactivamente con una oferta de productos, capaz de contrarrestar la enorme volatilidad de la fidelidad por parte de la demanda.

Los elementos antes expuestos, revelan que las empresas tienen el reto de sobrevivir, crecer, prosperar y ser rentables en un entorno turbulento por sus complejas relaciones, imponiéndoles operar con niveles de eficiencia, así como ágilmente a fin de construir cadenas de valor, eliminando barreras internas, para orientarse por entero a lo verdaderamente fundamental: sus relaciones con sus audiencias claves, es decir, con los clientes, socios, proveedores, trabajadores y el gobierno. En este contexto Hartman (2000, p. 11) dice que "Los clientes en la economía basada en Internet están bien informados y sus expectativas son cada vez mayores. De este modo, la capacidad de responder rápidamente a las demandas de los clientes, generando valor añadido, resulta imperativo".

Evidentemente el talento humano es la piedra angular para propiciar desde "nuevas lógicas" empresariales respuesta a las necesidades de cambios en el comportamiento de la relación binomial oferta-demanda en las instituciones dedicadas a la gestión y producción de servicio puro basado en las personas. Relación que desde la oferta Berry (1998, p. 9) se expresa en los siguientes términos:

La mayoría de los cargos de servicio tienen un alto contenido de criterio personal. La cantidad máxima de energía e interés que la persona pueda aportar a la función de servicio y la cantidad mínima requerida para evitar el castigo son bastante diferen- tes. Por lo tanto, los niveles de energía y atención del servicio dependen del criterio de quienes lo prestan.

En las empresas dedicadas a los servicios puros basados en las personas, los clientes conocen a muchos trabajadores y a menudo interactúan con ellos durante períodos prolongados. En consecuencia, la participación directa en la producción del servicio implica múltiples contactos con los consumidores o usuarios, quienes evalúan la calidad por la apariencia, habilidades sociales, conocimientos y capacidades técnicas de los trabajadores.

Los servicios asumen importancias significativas para la economía y la sociedad cada día. Según Camino (2005, p. 3) "El comercio internacional de servicios en el mundo representa aproximadamente un $17 \%$ del total de flujos considerando bienes, servicios, rentas y transferencias". En este mismo orden de ideas, Sáez (2008, p. 28) señala "Los países en desarrollo representaban alrededor de un $23 \%$ del comercio mundial de servicios en el 2002". Ahora bien, desde un punto de vista exclusivamente operativo, las acciones que implican los servicios pueden ser tangibles o intangibles.

Las primeras están dirigidas al cuerpo de las personas 0 a sus posesiones físicas, mientras las segundas, se dedican a la mente de las personas o sus activos intangibles, dando así, la naturaleza pura a los servicios cuando las acciones y actuaciones están destinadas a satisfacer necesidades, deseos, expectativas, incluso las fantasías dirigidas al cuerpo y la mente de las personas. (Lovelock et al., 2004, Zeithaml et al., 2002, Kotler et al., 2000, Berry, 1998, Cottle, 1990).

Un ejemplo del caso servicio puro basado en las personas, lo establece Kotler et al. (2004, p. 25) cuando dice: "en la mayoría de los servicios turísticos, tanto el suministrador del servicio como el cliente deben estar presentes para que la operación tenga lugar. Los empleados que atienden al cliente forman parte del producto". Desde esta óptica, es indiscutible que los servicios turísticos necesitan de gobernantes, inversionistas, empresarios, gerentes y trabajadores concienzudos, con ética sólida, agradables, 
expansivos, empáticos, asertivos, persuasivos, pero también motivados a captar, retener $y$ conservar a largo plazo las preferencias de los turistas por el destino turístico. En este sentido, se comparte la idea de Findlay (2001), citado por Sáez ob. cit. (p. 35) cuando expone:

Una estrategia de desarrollo del sector de servicios debe tener en cuenta la interacción que existe entre distintos sectores de servicios para obtener la máxima ganancia de los esfuerzos que se realizan en un sector y además la relación que puede existir entre los modos de suministro para el mayor aprovechamiento de las oportunidades.

En efecto, una estrategia para progresar con una oferta de exportación de servicios debería contemplar un mejoramiento del ambiente regulatorio que afecta el sector, favoreciendo el desarrollo local del emprendimiento, con factores claves que faciliten el crecimiento de las actividades, como refiere Vaillant (2008), citado por la Sáez ob. cit. (p. 35), "la disponibilidad de recursos humanos calificados, cierto desarrollo de infraestructura especializada (conectividad), estabilidad de los marcos jurídicos".

El prototipo de servicio puro reseñado por Kotler et al. (2004) es complementado por Findlay (2001), citado por Sáez Ídem cuando destaca:

Una política de promoción del sector turismo que no tome en cuenta la infraestructura de transporte, la seguridad de los turistas, las políticas migratorias y las limitaciones que puedan haber en el transporte de pasajeros en otros países (libertades en el tráfico aéreo) puede significar en definitiva en un menor impacto en el sector que una estrategia que aborde de manera global los determinantes del sector.

Para hacer realidad lo anterior, entre otras cosas, las empresas e instituciones dedicadas a los servicios turísticos se deben esmeran en captar, capacitar, desarrollar, motivar, remunerar y retener a los mejores trabajadores para sí. Porque de nada vale disponer de capacidades y recursos turísticos, si no se cuenta con un recurso humano altamente calificado para dar movimiento a las demás capacidades y recursos a disposición del destino turístico. Tal como sostiene Berry ob. cit. (p. 197) cuando expresa que "los servicios son ac- tuaciones, y las personas son los actores. Desde el punto de vista de los clientes, las personas que prestan el servicio son la compañía”.

Es evidente la importancia de la inducción, formación y desarrollo permanente del recurso humano en este tipo de empresas. En este sentido se comparte con Felipe y Felipe (1999, p. 43) cuando expresa que "El activo más importante de la empresa es la cantidad y calidad de CONOCIMIENTO a su disposición, de igual forma, cómo lo utiliza". Siguiendo a estos mismos autores, la empresa se ha convertido en la promotora del "aprendizaje permanente [...] en el siglo XXI; por esta razón, debe generar algo más, aparte de los beneficios monetarios, exigidos en la nueva sociedad del OCIO-TURISMO; en consecuencia, su responsabilidad es mayor al impulsar el crecimiento educacional y formativo de sus colaboradores".

La Organización Mundial del Turismo (OMT), organismo especializado de las Naciones Unidas en el campo del turismo, durante la decimotercera reunión de Asamblea General celebrada en Santiago de Chile del 27 de septiembre al 1 de octubre de 1999, proclamó solemnemente el Código Ético Mundial para el Turismo, el cual expresa en su Artículo 1: Contribución del turismo al entendimiento y al respeto mutuo entre hombres y sociedades; en su Numeral 3, señala:

Tanto las comunidades receptoras como los agentes profesionales locales habrán de aprender a conocer y a respetar a los turistas que los visitan, y a informarse sobre su forma de vida, sus gustos y sus expectativas. La educación y la formación que se impartan a los profesionales contribuirán a un recibimiento hospitalario de los turistas.

En su Artículo 9, Derechos de los trabajadores y de los empresarios del sector turístico, en el Numeral 2, se establece que:

Los trabajadores asalariados y autónomos del sector turístico y de las actividades conexas tienen el derecho y el deber de adquirir una formación inicial y continua adecuada. Se les asegurará una protección social suficiente y se limitará en todo lo posible la precariedad de su empleo. Se propondrá un estatuto particular a los trabajadores estacionales del sector, 
especialmente en lo que respecta a su protección social.

En los artículos precedente del Código Ético Mundial para el Turismo, se reconoce explícitamente la necesidad de educar, capacitar, formar y desarrollar profesionalmente a los trabajadores dedicados a la actividad turística, como una acción para disponer de una oferta de trabajadores calificados y capaces de generar beneficios a largo plazo para la persona misma y el resto de la sociedad, a través del aumento de la productividad laboral de los individuos.

Por su parte, la Organización Internacional del Trabajo (OIT) está consagrada a la promoción de oportunidades de trabajo decente y productivo para mujeres y hombres, en condiciones de libertad, igualdad, seguridad y dignidad humana. Sus objetivos principales son promover los derechos laborales, fomentar oportunidades de empleo dignas, mejorar la protección social y fortalecer el diálogo al abordar temas relacionados con el trabajo.

El desarrollo de los recursos humanos, en particular la educación y la formación, son fundamentales para la consecución del objetivo de la OIT de crear mayores oportunidades tanto para las mujeres como a los hombres de obtener un trabajo decente y al mismo tiempo productivo. Visto de esta forma, tanto la educación como la formación son aspectos centrales del Programa de Trabajo Decente. En este sentido la Conferencia General de la OIT, convocada en Ginebra por su Consejo de Administración el 1 de junio de 2004, en su nonagésima segunda reunión y después de un amplio debate aprobaron la Recomendación 195, sobre el desarrollo de los recursos humanos: educación, formación y aprendizaje permanente. Se trata de una norma internacional del trabajo de la OIT de carácter dinámico, completa en su formulación que ayudará a los gobiernos, empleadores y trabajadores a poner en práctica sistemas integrales de educación, formación y aprendizaje permanente para el siglo XXI.

La Recomendación 195, ubica a las personas en un lugar central del desarrollo económico y social. En ella se exhorta a los estados miembros a formular e implementar políticas de educación, formación y aprendizaje permanente que promuevan la empleabilidad de las personas a lo largo de la vida. La mejor manera de elaborar estas políticas es a través de un proceso de diálogo social.
Para resultar eficaces, las políticas han de formar parte sustancial e integrales en los ámbitos económico, social, mercado de trabajo y de igual manera en los programas destinados al crecimiento económico, incluyendo el empleo. La educación de calidad, la formación previa al empleo y el aprendizaje a lo largo de la vida son los tres pilares necesarios para fomentar, con la debida protección, la empleabilidad de las personas, garantizada por mecanismos transparentes e imparciales de evaluación, certificación y reconocimiento de que las aptitudes profesionales adquiridas son transferibles en las empresas, industrias e instituciones educativas. Como alternativa para superar los retos que impone el mundo laboral futuro, tal como refiere Ibarra (2000, pp. 96-97) cuando expresa:

Por su parte, los principales cambios en los procesos productivos se refieren al tránsito de una producción en serie, a una producción diferenciada; de la especialización del trabajo, a la polivalencia y la multifuncionalidad; del puesto de trabajo, a la organización en redes y equipos autodirigidos; $y$, de las actividades repetitivas y rutinarias, a la innovación y creatividad del trabajador para participar en el análisis y solución de problemas relacionados con la calidad, así como en la interacción con otros trabajadores y en la toma de decisiones. De esta manera se ha generado una nueva estructura industrial en el mundo, caracterizada por la "descorporatización" o núcleos de negocios capaces de innovar y adaptarse para responder a las cambiantes demandas del mercado.

Ibarra (2000), al declarar lo antes citado, dejó claro que los trabajadores del nuevo contexto de los negocios deben emprender permanentemente la formación y el desarrollo de competencias laborales para adecuarse a las exigencias presente y futuras del mercado laboral, como acción para garantizar su empleabilidad y competitividad de los negocios.

El desarrollo, formación y aprendizaje permanentes se debe contemplar como una actividad estratégica continua en la fuerza de trabajo de las empresas hoteleras para adaptarse con flexibilidad a los cambios técnicos, sociales y económicos a fin de lograr la eficiencia, eficacia, productividad y calidad de los servicios ofertados por los establecimientos hoteleros inscritos en el RTN y categorizados como hoteles cinco estrellas en la Isla de Margarita. 
Además, se ha observado decenas de gerentes en acción, percibiéndose que en la mayoría de los establecimientos hoteleros de cinco estrellas, carecen en la actualidad de la conducción requerida en las diferentes posiciones gerenciales; no significa esto, que quienes ocupan tales puestos de trabajo sean trabajadores sin energía y talento. Todo lo contrario, se trata de gente laboriosa, brillante, con experiencia, casi todos tratando de hacer lo que consideran correcto. Sin embargo, no logran inspirar, entusiasmar y comprometer de manera sostenible a la fuerza de trabajo y a los huéspedes interesados en ser escuchados para responder acertadamente a los cambios, a fin de garantizar la supervivencia, crecimiento, rentabilidad y prosperidad de las empresas.

En este sentido, se comparte con Drucker (2002, p. 85) quien vaticinó "El director ejecutivo de mañana tendrá que aprender cuándo ordenar y cuándo actuar como socio". Este notable experto en gerencia, deslumbró acerca de los cambios como una constante y la forma como los gerentes en todas las instancias de los negocios podrían gestionarlos para satisfacer las necesidades, deseos, expectativas y fantasías tan complejas, variando casi instantáneamente.

Con este planteamiento, la investigación se propuso como objetivo secuencial diagnosticar las necesidades de formación y desarrollo de competencias gerenciales en el talento humano, a nivel gerencial, de los establecimientos hoteleros inscritos en el RTN y categorizados como hoteles cinco estrellas en la Isla de Margarita.

\section{Metodología}

Desde la dimensión temporal, la investigación planteada es de tipo transeccional o transversal porque se recogió la información a través del instrumento aplicado a una muestra representativa de la población de trabajadores a nivel de gerencia de los hoteles categorizados como cinco estrellas e inscritos en el RTN de una sola vez y durante un período de corta duración comprendido entre los meses de diciembre 2010 y julio 2011 y desde la dimensión nivel del conocimiento, el estudio se abordó como una investigación aplicada y analítica al comparar las relaciones subyacentes entre las dimensiones de las variables objeto de estudio.

En atención al objetivo propuesto, la investigación se orientó hacia la incorporación de un diseño de estudio de campo, donde la misma estuvo sustentada en la delimitación de los hechos concretos que no sólo permitió la observación sino también la recolección de los datos directamente de la realidad objeto de estudio, en su ambiente cotidiano para luego realizar el análisis, interpretación y presentación de los resultados de las indagaciones (Padrón, 2006).

Geográficamente el área de estudio se circunscribió al estado Nueva Esparta, específicamente en la Isla de Margarita, en los Municipios Autónomos Gómez, Maneiro y Mariño respectivamente. La investigación se realizó en las empresas de alojamiento turísticos categorizadas como hoteles cinco estrellas e inscrita legal y formalmente en el RTN que mantiene el Ministerio para el Poder Popular del Turismo en Venezuela.

La población que se estudió fue finita y se encuentra referida a todos los trabajadores a nivel de gerencial, entre los meses de diciembre 2010 hasta julio 2011. La misma estuvo conformada por 269 trabajadores distribuidos en seis hoteles y 19 puestos de trabajos. La población objeto de estudio representa $13,1 \%$ del total de la fuerza de trabajo de los establecimientos hoteleros inscritos en el RTN y categorizados como hoteles cinco estrellas en la Isla de Margarita. La muestra estadística, se determinó a través de un muestreo aleatorio simple, con un nivel de confianza de $95,5 \%$, un error estándar del $10 \%$ y a dos sigmas, sin reemplazo con afijación proporcional, conformándola 71 unidades muestrales (Mendenhal, 1990).

La información de primera mano se recopiló por medio de un cuestionario revisado por siete expertos, lo que llevó a la eliminación de algunos ítems y a la modificación de la redacción de algunos otros; el cuestionario final quedó formado por 15 ítems, concentrados en la identificación de las necesidades formación y desarrollo de las competencias del referido talento humano. Los ítems se midieron mediante la escala Likert de cinco puntos, que variaran desde 1 (máximo desacuerdo) a 5 (máximo acuerdo).

La idea principal fue ofrecer un conjunto de ítems presentados en forma de afirmaciones con las cinco alternativas de respuestas al interlocutor quien debió escoger, solamente entre ellas, aquella igual o más semejante a su propia forma 
de respuesta, considerando como dato inválido a quien marque dos o más opciones.

Una vez cerrado el proceso de recepción de cuestionarios, se procedió a la informatización de los datos mediante la creación de una base de datos y su posterior tratamiento estadístico descriptivo, haciendo uso del paquete estadístico SPSS V 15.0 (Statistical Package for Social Sciences), cuyo propósito principal fue definir la estructura subyacente de la serie de datos que permitió analizar las interrelaciones que existe entre las variables, factores y componentes principales, para esbozar los hechos y acciones concretas de la realidad objeto de estudio.

En lo referente al trabajo de campo, se adoptó una metodología de contacto, entrega y seguimiento de los cuestionarios. Se contactó telefónicamente con los gerentes generales y de recursos humanos de los hoteles objetos de estudio, solicitándoles participar en el estudio así como su utilidad, comprometiéndose el investigador a enviarle los resultados de la investigación, si así lo requerían. También se aseguró la confidencialidad, globalidad y anonimato durante el análisis de los datos.

\section{Resultados y discusión}

Las competencias se han utilizado como estrategia empresarial exitosa, desde comienzos de la década de 1970 cuando McClelland (1973), aplicó por primera vez el concepto. En este orden de ideas, para Boyarzis (1982), citado por Fernández, Cubeiro y Dalziel (1996, p. 28) las competencias son: "Los conjuntos de habilidades, conocimientos, aptitudes, características de conducta y otros atributos que en la combinación correcta y en la situación debida, predicen un rendimiento superior".

Desde este enfoque, las necesidades de formación y desarrollo de competencias gerenciales, se agruparon por indicadores de la siguiente manera:

La formación realizada en el hotel para alcanzar un nivel de actividad física apropiado a fin de cumplir las tareas, cometidos, actividades y responsabilidades gerenciales en jornadas de trabajo prolongadas, arrojó $57,7 \%$ de opiniones favorables; analizado de manera particular, indica que $33,8 \%$ de los trabajadores gerenciales se ubican en la categoría "de acuerdo", mientras $23,9 \%$ se encuentra "totalmente de acuerdo"; sin embargo, existe $42,3 \%$ de opiniones desfavorable al indicador incluyendo a los indecisos. Este último resultado se interpreta como una necesidades de formación para la muestra estudiada (Tabla 1), es decir $42,3 \%$ de la muestra analiza necesita formación para alcanzar un nivel de actividad física apropiado en la función gerencial.

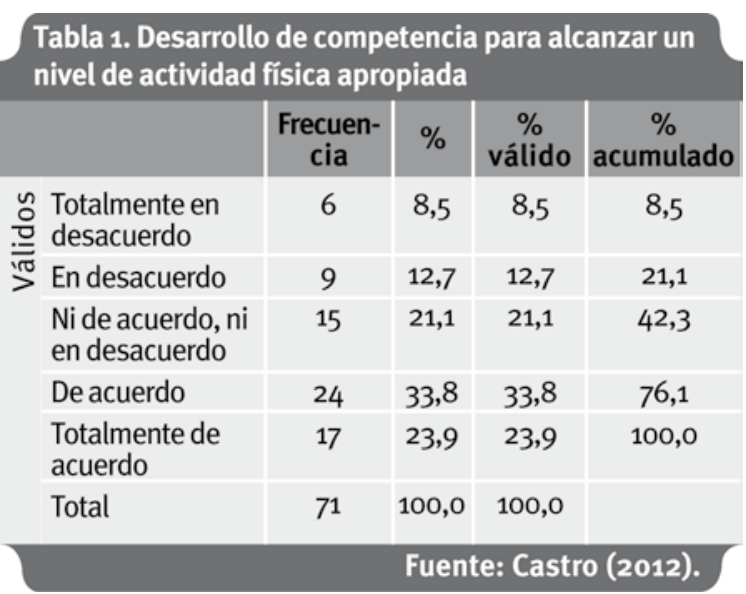

Las actividades de formación realizada en el hotel para tomar iniciativas ante las dificultades que plantea la función gerencial, logró $64,8 \%$ de opiniones favorables, desglosas en $43,7 \%$ para la categoría "de acuerdo" y $21,1 \%$ "totalmente de acuerdo" (Tabla 2); en contraposición 35,2\% manifestaron respuestas negativas al indicador, interpretándose como una necesidades de formación para desarrollar en el gerente la competencia que permita tomar iniciativas rápidas ante las pequeñas dificultades o problemas que surgen en el día a día de las actividades.

\begin{tabular}{|c|c|c|c|c|}
\hline & $\begin{array}{l}\text { Frecuen- } \\
\text { cia }\end{array}$ & $\%$ & $\begin{array}{c}\% \\
\text { válido }\end{array}$ & $\begin{array}{c}\% \\
\text { acumulado }\end{array}$ \\
\hline $\begin{array}{l}\text { 乞 Totalmente en } \\
\text { 을 desacuerdo }\end{array}$ & 5 & 7,0 & 7,0 & 7,0 \\
\hline 㲾 En desacuerdo & 11 & 15,5 & 15,5 & 22,5 \\
\hline $\begin{array}{l}\text { Ni de acuerdo, ni } \\
\text { en desacuerdo }\end{array}$ & 9 & 12,7 & 12,7 & 35,2 \\
\hline De acuerdo & 31 & 43,7 & 43,7 & 78,9 \\
\hline $\begin{array}{l}\text { Totalmente de } \\
\text { acuerdo }\end{array}$ & 15 & 21,1 & 21,1 & 100,0 \\
\hline Total & 71 & 100,0 & 100,0 & \\
\hline
\end{tabular}

La opinión arrojada por los gerentes acerca de la formación realizada en el hotel para percibir la urgencia real de determinadas tareas, cometidos, actividades y responsabilidades gerenciales, midió $45,1 \%$ de respuestas favorables, 
desagregadas en $36,6 \%$ para la categoría "ee acuerdo) y 8,5\% "totalmente de acuerdo" (Tabla 3 ); en oposición se registró $54,9 \%$ de opiniones desfavorable, descifrándose como una necesidad de formación para desarrollar la competencia para percibir las urgencias reales en la función gerencial, coordinando las acciones de varias partes del cuerpo para ponerse en movimiento con celeridad, porque el tiempo apremia, con el fin de alcanzar la realización de este propósito en plazos muy breves.

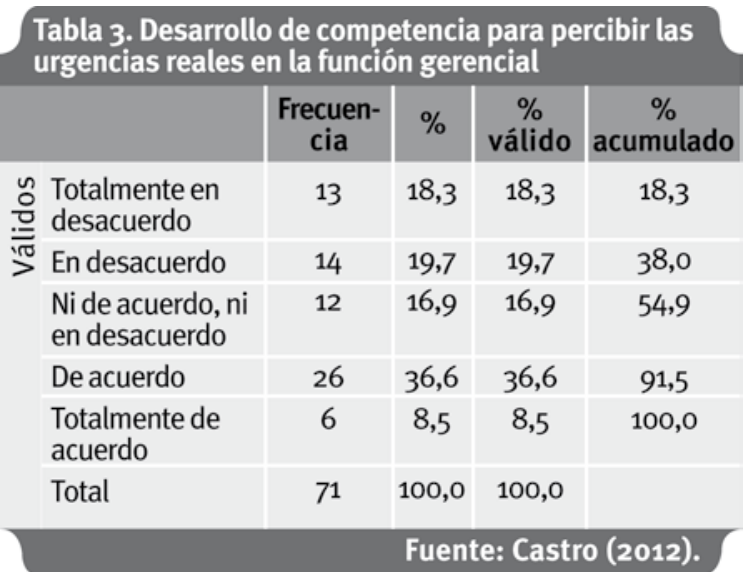

El indicador de formación realizada en el hotel para analizar e interpretar rápida y correctamente datos numéricos, captó $33,8 \%$ de opiniones a favor, adjudicándose en la categoría "de acuerdo" $28,2 \%$ y $5,6 \%$ "totalmente de acuerdo". Mientras que $66,2 \%$ opinaron de manera desfavorable al indicador, deduciéndose así la necesidad de formación para desarrollar la competencia que permita a los gerentes analizar e interpretar rápida y correctamente datos numéricos, discerniendo de lo fundamental sobre lo superfluo, para establecer conexiones relevantes.

El indicador formación realizada en el hotel para la comprensión y fluidez a fin de comunicarse correctamente en forma verbal y escrita en otros idiomas, registró $38,1 \%$ de opiniones favorables;, analizado de manera particular, indica que $25,4 \%$ de la muestra se encuentra en la categoría "de acuerdo" y 12,7\% "totalmente de acuerdo" (Tabla 4), mientras que en oposición registró $61,9 \%$ opiniones negativas, interpretándose este resultado como una necesidad de formación para desarrollar la competencia que facilite a los gerentes, la comprensión, el dominio y la fluidez para comunicarse correctamente en forma verbal y escrita con los huéspedes, clientes, trabajadores y relacionados en los idiomas inglés, alemán, francés y español.
Tabla 4. Desarrollo de competencia para la comprensión y fluidez a fin de comunicarse correctamente en forma verbaly escrita en otros idiomas

\begin{tabular}{|c|c|c|c|c|}
\hline & $\begin{array}{l}\text { Frecuen- } \\
\text { cia }\end{array}$ & $\%$ & $\begin{array}{c}\% \\
\text { válido }\end{array}$ & $\begin{array}{c}\% \\
\text { acumulado }\end{array}$ \\
\hline $\begin{array}{l}\text { Totalmente en } \\
\text { desacuerdo }\end{array}$ & 12 & 16,9 & 16,9 & 16,9 \\
\hline En desacuerdo & 18 & 25,4 & 25,4 & 42,3 \\
\hline $\begin{array}{l}\text { Ni de acuerdo, ni } \\
\text { en desacuerdo }\end{array}$ & 14 & 19,7 & 19,7 & 62,0 \\
\hline De acuerdo & 18 & 25,4 & 25,4 & 87,3 \\
\hline $\begin{array}{l}\text { Totalmente de } \\
\text { acuerdo }\end{array}$ & 9 & 12,7 & 12,7 & 100,0 \\
\hline Total & 71 & 100,0 & 100,0 & \\
\hline
\end{tabular}

El indicador formación realizada en el hotel para operar de manera rápida y sencilla las tecnologías de información y comunicación (TIC), midió $45,1 \%$ de resultados positivos, desagregados en 32,4\% para la categoría "de acuerdo" y $12,7 \%$ "totalmente de acuerdo" (Tabla 5), en contradicción $54,9 \%$ de las mediciones resultaron negativas para el indicador, dato que se deduce como una necesidad de formación para desarrollar en la muestra investigada la competencia para operar de manera rápida y sencilla las TIC, incluyendo sus aparatos y programas para procesar, integrar y transmitir información a los clientes internos y externos del hotel, manteniendo interacción en tiempo real a través de la Internet, Intranet, Extranet y las aplicaciones ofimáticas entre otras.

\begin{tabular}{|c|c|c|c|c|}
\hline & $\begin{array}{l}\text { Frecuen- } \\
\quad \text { cia }\end{array}$ & $\%$ & $\begin{array}{c}\% \\
\text { válido }\end{array}$ & $\begin{array}{c}\% \\
\text { acumulado }\end{array}$ \\
\hline 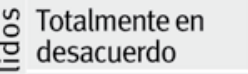 & 17 & 23,9 & 23,9 & 23,9 \\
\hline En desacuerdo & 10 & 14,1 & 14,1 & 38,0 \\
\hline $\begin{array}{l}\text { Ni de acuerdo, ni } \\
\text { en desacuerdo }\end{array}$ & 12 & 16,9 & 16,9 & 54,9 \\
\hline De acuerdo & 23 & 32,4 & 32,4 & 87,3 \\
\hline $\begin{array}{l}\text { Totalmente de } \\
\text { acuerdo }\end{array}$ & 9 & 12,7 & 12,7 & 100,0 \\
\hline Total & 71 & 100,0 & 100,0 & \\
\hline & & \multicolumn{3}{|c|}{ Fuente: Castro (2012). } \\
\hline
\end{tabular}

El indicador formación realizada en el hotel para desarrollar competencia a fin de analizar el entorno del negocio hotelero, asegurando la supervivencia, crecimiento, rentabilidad, productividad y competitividad, cuantificó $43,7 \%$ de opiniones favorable, descomponiéndose en $25,4 \%$ para la categoría "de acuerdo" y $18,3 \%$ "totalmente de acuerdo" (Tabla 6), contraria- 
mente se registró $56,3 \%$ de datos desfavorables, interpretándose este resultado como una necesidad de formación para desarrollar en la muestra estudiada la competencia para reconocer los peligros y aprovechar las oportunidades del entorno hotelero que repercuten en la supervivencia, crecimiento, rentabilidad, productividad y competitividad del negocio, haciendo aportes concretos de acuerdo con su campo de responsabilidad para la formulación, implantación y evaluación de la estrategia a corto, mediano y largo plazo.

\begin{tabular}{|c|c|c|c|c|}
\hline & $\begin{array}{l}\text { Frecuen- } \\
\quad \text { cia }\end{array}$ & $\%$ & $\begin{array}{l}\% \\
\text { válido }\end{array}$ & $\begin{array}{c}\% \\
\text { acumulado }\end{array}$ \\
\hline $\begin{array}{l}\text { 을 Totalmente en } \\
\text { 으 desacuerdo }\end{array}$ & 12 & 16,9 & 16,9 & 16,9 \\
\hline 的 En desacuerdo & 15 & 21,1 & 21,1 & 38,0 \\
\hline $\begin{array}{l}\text { Ni de acuerdo, ni } \\
\text { en desacuerdo }\end{array}$ & 13 & 18,3 & 18,3 & 56,3 \\
\hline De acuerdo & 18 & 25,4 & 25,4 & 81,7 \\
\hline $\begin{array}{l}\text { Totalmente de } \\
\text { acuerdo }\end{array}$ & 13 & 18,3 & 18,3 & 100,0 \\
\hline Total & 71 & 100,0 & 100,0 & \\
\hline
\end{tabular}

En los hoteles estudiados, la formación para lograr establecer relaciones interpersonales, superando conflictos emocionales, midió $52,1 \%$ de opiniones positivas; en oposición se observó $47,9 \%$ de resultados desfavorables a la afirmación del indicador, arrojando este dato una necesidad de formación para desarrollar en los gerentes de los hoteles cinco estrellas la competencia para establecer relaciones interpersonales, superando conflictos emocionales, para expresarse y cooperar abiertamente a pesar de la oposición del resto del equipo, e incluso de forma anónima, en la consecución de objetivos comunes, subordinando los propios intereses a los intereses colectivos y considerando como más relevante el objetivo de todos que las circunstancias personales que se han de sacrificar o posponer.

Tratándose de empresas de servicios puros, donde las interacciones humanas son significativas para alcanzar experiencias memorables tanto para huéspedes como en los trabajadores, resulta contradictorio que se manifieste abiertamente tal necesidad de formación. Se trata de una conducta importante para los gerentes; tal como señala Ansorena (1997, p. 191) "Es la capacidad de mostrar que se ha entendido de manera profunda los sentimientos y el estado emocional de los demás, y que se es consciente de hasta dónde se puede actuar sobre ello".

Las actividades de formación realizada en el hotel para desarrollar comportamientos a fin de alcanzar la gestión conscientemente de los recursos y capacidades, cuantificó $43,7 \%$ de resultados positivos para la afirmación, descomponiéndose por categoría en un $31 \%$ "de acuerdo" y 12,7\% "totalmente de acuerdo" (Tabla 7), contra $56,3 \%$ de opiniones negativas. Este resultado evidencia una necesidad de formación para desarrollar en la muestra estudiada la competencia a fin de ejecutar políticas, acciones y decisiones que impliquen la utilización consciente de los recursos materiales, económicos, humanos, tecnológicos e inmateriales de modo rápido y económico combinando eficiencia y eficacia para conseguir los objetivos organizacionales.

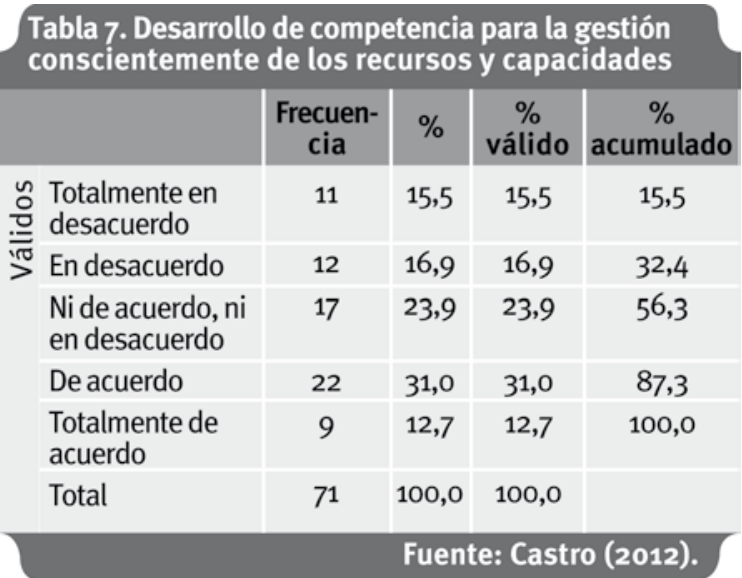

Por su parte la formación realizada en el hotel para alcanzar actuaciones encaminadas a satisfacer las necesidades de la demanda, registró $74,7 \%$ de opiniones favorables; analizado el dato de forma particular, indica que $47,9 \%$ de la muestra se encuentra "de acuerdo" y $26,8 \%$ "totalmente de acuerdo" (Tabla 8 ), en oposición se midió $25,3 \%$ de opiniones desfavorables.

A pesar que este indicador cuantificó uno de los resultados más alto, se considera conveniente reconocer la necesidad de formación para reforzar el desarrollo de la competencia que facilite alcanzar comportamientos encaminados a satisfacer las necesidades, deseos, expectativas y fantasías de la demanda, superando sus expectativas con ofertas que agreguen valor, cuidando minuciosamente 
todos los detalles de la relación, procurando eliminar errores y duplicidades, así como, proporcionando respuestas acertadas y oportunas a las peticiones y sugerencias de los huéspedes, en virtud de que la demanda es cada días más exigente.

El indicador formación realizada en el hotel para mantener y utilizar una amplia red de relaciones con personas claves en la empresa y del sector turístico hotelero, obtuvo $55 \%$ de opiniones positivas, descompuestas en la categoría "de acuerdo" con 42,3\% y 12,7\% "totalmente de acuerdo" (Tabla 9); contrariamente $45 \%$ de la muestra opinó negativamente con respecto al indicador, interpretándose este resultado como una necesidad de formación para desarrollar, la competencia para crear, mantener y utilizar una amplia red de relaciones con personas claves en la empresa y del sector turístico hotelero, estando informado, dentro de una prudencia y economía de tiempo y esfuerzo, en líneas generales de los sucesos importantes que ocurren en el entorno y que afectan al negocio, al sector hotelero, a la actividad turística y a la estrategia del hotel.

\begin{tabular}{|c|c|c|c|c|}
\hline & $\begin{array}{l}\text { Frecuen- } \\
\text { cia }\end{array}$ & $\%$ & $\begin{array}{c}\% \\
\text { válido }\end{array}$ & $\begin{array}{c}\% \\
\text { acumulado }\end{array}$ \\
\hline $\begin{array}{l}\text { ¿ } \text { Totalmente en } \\
\text { :o desacuerdo }\end{array}$ & 2 & 2,8 & 2,8 & 2,8 \\
\hline En desacuerdo & 11 & 15,5 & 15,5 & 18,3 \\
\hline $\begin{array}{l}\text { Ni de acuerdo, ni } \\
\text { en desacuerdo }\end{array}$ & 5 & 7,0 & 7,0 & 25,4 \\
\hline De acuerdo & 34 & 47,9 & 47,9 & 73,2 \\
\hline $\begin{array}{l}\text { Totalmente de } \\
\text { acuerdo }\end{array}$ & 19 & 26,8 & 26,8 & 100,0 \\
\hline Total & 71 & 100,0 & 100,0 & \\
\hline
\end{tabular}

Tabla 9. Desarrollo de competencia para mantener y utilizar una amplia red de relaciones con personas claves en la empresa y del sector turístico hotelero

\begin{tabular}{|c|c|c|c|c|}
\hline & $\begin{array}{l}\text { Frecuen- } \\
\text { cia }\end{array}$ & $\%$ & $\begin{array}{c}\% \\
\text { válido }\end{array}$ & $\begin{array}{c}\% \\
\text { acumulado }\end{array}$ \\
\hline $\begin{array}{l}\text { 으 Totalmente en } \\
\text { 으 desacuerdo }\end{array}$ & 5 & 7,0 & 7,0 & 7,0 \\
\hline En desacuerdo & 11 & 15,5 & 15,5 & 22,5 \\
\hline $\begin{array}{l}\text { Ni de acuerdo, ni } \\
\text { en desacuerdo }\end{array}$ & 16 & 22,5 & 22,5 & 45,1 \\
\hline De acuerdo & 30 & 42,3 & 42,3 & 87,3 \\
\hline $\begin{array}{l}\text { Totalmente de } \\
\text { acuerdo }\end{array}$ & 9 & 12,7 & 12,7 & 100,0 \\
\hline Total & 71 & 100,0 & 100,0 & \\
\hline
\end{tabular}

Fuente: Castro (2012).
El indicador formación realizada en el hotel para fijar metas personales y laborales, midió $71,8 \%$ de opiniones positivas, distribuidas por categoría en $47,9 \%$ "de acuerdo" y $23,9 \%$ "totalmente de acuerdo"; en oposición se registró $28,2 \%$ de resultados negativos al indicador; no obstante, al alto número de respuestas satisfactorias, es prudente mantener activa la necesidad de capacitación para desarrollar en la muestra investigada, la competencia que permita establecer metas personales y laborales, a la vez que propicie la consecución de resultados positivos tanto propios como organizacionales, aún más allá de las exigencias institucionales o de la línea jerárquica, mostrando un alto impulso para conseguir retos y desafíos personales y profesionales, aplicando de forma autodirigida la creatividad y originalidad de planteamientos novedosos para alcanzar las metas.

El indicador formación realizada en el hotel para expresar pensamientos o contenidos internos de manera comprensible al interlocutor, captó $70,4 \%$ de respuestas favorables, correspondiendo $46,5 \%$ a la categoría "de acuerdo" y $23,9 \%$ "totalmente de acuerdo"; en oposición $29,6 \%$ de los resultados arrojados son negativo para el indicador, descifrándose con este dato que persiste una necesidad de formación que permita desarrollar en la muestra estudiada la competencia para expresar pensamientos o contenidos internos de manera comprensible al interlocutor, cara a cara, con toda la potencialidad de la palabra hablada, utilizada de forma proporcional al objetivo y a la audiencia que recibe el mensaje, aprovechando las imágenes verbales y los recursos lingüísticos adecuados, a la vez que mantiene el diálogo controlado.

El indicador formación realizada en el hotel para guiar, inspirar, entusiasmar, persuadir y dirigir las acciones de grupos de personas, midió $47,9 \%$ de respuestas positivas, desagregándose en 36,6\% para la categoría "de acuerdo" y 11,3\% "totalmente de acuerdo" (Tabla 10); contrariamente más de la mitad $(52,1 \%)$ de la muestra analizada reportó negativamente el indicador, interpretándose este dato como una necesidad de formación que tienen los gerentes para guiar, inspirar, entusiasmar, persuadir, dirigir las acciones y anticipar los posibles escenarios de grupos de personas y estableciendo, manteniendo el espíritu de grupo para alcanzar los objetivos del mismo. 
Tabla 10. Desarrollo de competencia para guiar, inspirar, entusiasmar, persuadir y dirigir las acciones de grupos de personas

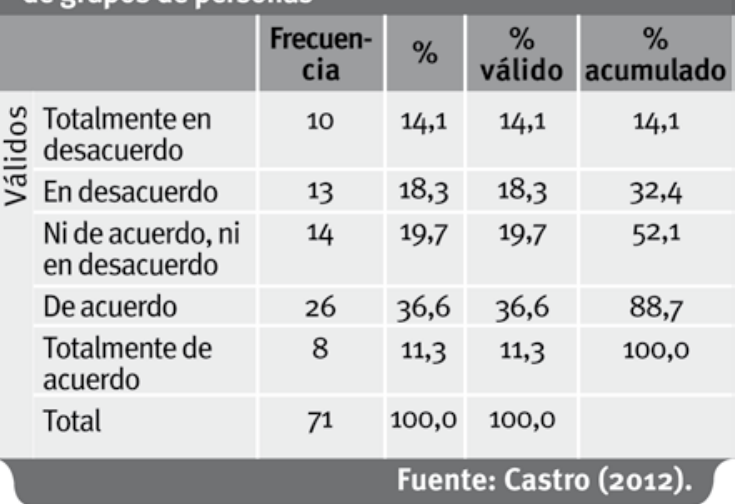

El indicador formación realizada en el hotel para lograr el compromiso de los trabajadores alcanzó $42,3 \%$ de respuestas favorables; menos de la mitad, adjudicándose $(32,4 \%)$ en la categoría "de acuerdo" y 9,9\% "totalmente de acuerdo" (Tabla 11); de manera opuesta $52,1 \%$ de las respuestas de la muestra, fueron desfavorables con respecto al indicador, analizándose este resultado como una necesidad de formación, para desarrollar en los gerente la competencia que permita lograr el compromiso de los trabajadores, orientándolos adecuada y eficazmente, inspirándolos en su confianza, dándole sentido a su trabajo y motivándoles a conseguir sus objetivos, a la vez que detecta y anticipa los problemas y dificultades que tienen los trabajadores en el desempeño de sus tareas, cometidos, actividades y responsabilidades para dotarlos de las capacidades y recursos necesarios con el propósito de mejorar su actuación.

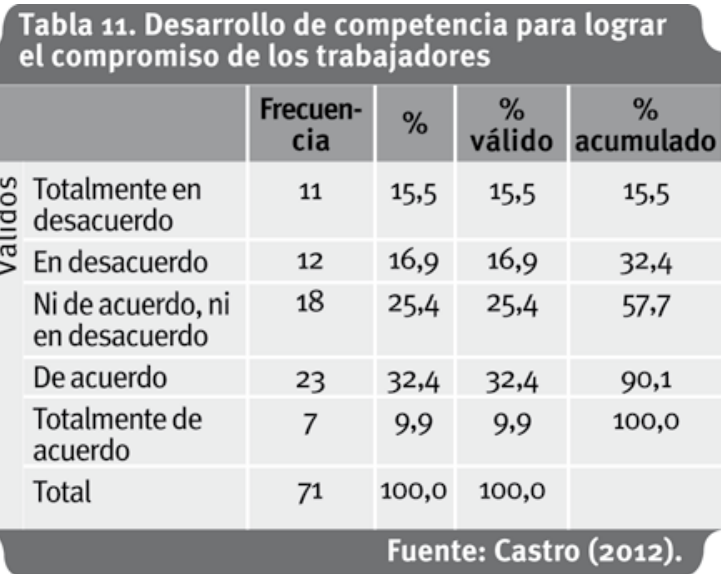

En términos generales, se puede afirmar que existen necesidades de formación para el desarrollo de competencias gerenciales de la fuerza de trabajo a nivel gerencial en los hoteles cinco estrellas de la Isla de Margarita; las mismas, son priorizadas considerando la frecuencia porcentual de las respuestas negativas a las afirmaciones de los indicadores (Tabla 12).

Tabla 12. Prioridad de las necesidades de formación para el desarrollo de competencias gerenciales en el talento humano de los hoteles cinco estrellas de la Isla de Margarita

\begin{tabular}{|c|c|c|}
\hline Prioridad & Necesidad de formación & $\%$ necesidad \\
\hline 1 & Desarrollo de competencia para analizar e interpretar rápida y correctamente datos numéricos & 66,2 \\
\hline 2 & $\begin{array}{l}\text { Desarrollo de competencia para la comprensión y fluidez a fin de comunicarse correctamente en forma } \\
\text { verbal y escrita en otros idiomas }\end{array}$ & 61,9 \\
\hline \multirow[t]{3}{*}{3} & $\begin{array}{l}\text { Desarrollo de competencia para analizar el entorno del negocio hotelero asegurando la supervivencia, } \\
\text { crecimiento, rentabilidad, productividad y competitividad }\end{array}$ & \multirow[t]{3}{*}{56,3} \\
\hline & $\begin{array}{l}\text { Desarrollo de competencia para establecer relaciones interpersonales, superando conflictos } \\
\text { emocionales }\end{array}$ & \\
\hline & Desarrollo de competencia para la gestión conscientemente de los recursos y capacidades & \\
\hline \multirow[t]{2}{*}{4} & Desarrollo de competencia para percibir las urgencias reales en la función gerencial & \multirow[t]{2}{*}{54,9} \\
\hline & $\begin{array}{l}\text { Desarrollo de competencia para opera de manera rápida y sencilla las tecnologías de información y } \\
\text { comunicación (TIC) }\end{array}$ & \\
\hline \multirow[t]{2}{*}{5} & $\begin{array}{l}\text { Desarrollo de competencia para guiar, inspirar, entusiasmar, persuadir y dirigir las acciones de grupos } \\
\text { de personas }\end{array}$ & \multirow[t]{2}{*}{52,1} \\
\hline & Desarrollo de competencia para lograr el compromiso de los trabajadores & \\
\hline 6 & $\begin{array}{l}\text { Desarrollo de competencia para mantener y utilizar una amplia red de relaciones con personas claves en } \\
\text { la empresa y del sector turístico hotelero }\end{array}$ & 45,0 \\
\hline 7 & Desarrollo de competencia para alcanzar un nivel de actividad física apropiada & 42,3 \\
\hline 8 & Desarrollo de competencia para tomar iniciativas ante las dificultades que plantea la función gerencial & 35,2 \\
\hline 9 & $\begin{array}{l}\text { Desarrollo de competencia para expresar pensamientos o contenidos internos de manera comprensible } \\
\text { para el interlocutor }\end{array}$ & 29,6 \\
\hline 10 & Desarrollo de competencia para fijar metas personales y laborales & 28,2 \\
\hline 11 & $\begin{array}{l}\text { Desarrollo de competencia para alcanzar comportamientos encaminados a satisfacer las necesidades } \\
\text { de la demanda }\end{array}$ & 25,3 \\
\hline
\end{tabular}




\section{Conclusiones}

Con respecto a la formación y desarrollo de competencias gerenciales, los resultados demuestran que existe la necesidad de emprender de forma inmediata la formación para el desarrollo de las competencias gerenciales para: 1) analizar e interpretar rápida y correctamente los datos numéricos; 2) alcanzar la comprensión y fluidez a fin de comunicarse correctamente en forma verbal y escrita en otros idiomas; 3) analizar el entorno del negocio hotelero asegurando la supervivencia, crecimiento, rentabilidad, productividad y competitividad; 4) establecer relaciones interpersonales, superando conflictos emocionales; $y, 5)$ gestionar conscientemente los recursos y capacidades por parte de los gestores en los hoteles cinco estrellas de la Isla de Margarita, porque las misma presentan opiniones desfavorables por parte de la muestra analizada por encima del $50 \%$.

\section{Recomendaciones}

Realizar una investigación para analizar la forma de aprovechar los incentivos tributarios establecidos en la legislación venezolana para financiar las actividades de formación y desarrollo de las competencias gerenciales. Asimismo, diseñar las actividades de formación considerando las características del perfil de la fuerza de trabajo; del mismo modo, planificar las actividades de formación y desarrollo de las competencias atendiendo la prioridad identificada en este estudio. Finalmente, se plantea desarrollar alianzas de cooperación con instituciones de educación en materia de hotelería de la Isla de Margarita.

\section{Referencias}

Ansorena, Á. (1997). 15 pasos para la selección del personal con éxito. Método e instrumentos. Barcelona, España: Paidós (p. 191).

Berry, L. (1998). ¡Un buen servicio ya no basta! Cuatro principios del servicio excepcional al clienta. ( $3^{\text {ra }}$ reimpresión de la $1^{\text {ra }}$ edición; de Hassan, A. Trad). Bogotá, Colombia: Norma (p. 9-197).

Camino, C. (2005). Comercio Internacional de Servicios en América Latina y el Caribe. Recuperado 21/05/2008 de: http://www.cepal. org/publicaciones/xml/9/23489/lcw46e.pdf (p. 3).
Cottle, D. (1990) El servicio centrado en el cliente. Cómo lograr que regresen y sigan utilizando sus servicios. ( $1^{\text {ra }}$ reimpresión de la $1^{\text {ra }}$ edición; Soriano, C. Trad.). Madrid, España: Diez de Santos.

Drucker, P. (2002). La gerencia en la sociedad futura. $\left(^{\text {ra }}\right.$ reimpresión de la $1^{\text {ra }}$ edición; Cárdenas, J. Trad.). Bogotá, Colombia: Norma (p. 85).

Felipe, J. y Felipe, I. (1999). Los nuevos perfiles profesionales de los directivos hoteleros. Madrid, España: Alción (p. 43).

Fernández, G., Cubeiro, J. y Dalziel, M. (1996). Las competencias: Clave para una gestión integrada de los recursos humanos ( $2^{\mathrm{da}}$ ed.). Bilbao, España: Deusto. (p. 28).

Hartman, A., Sifonis, J. y Kador, J. (2000). Net Ready Estrategias para el éxito en la nueva economía. Madrid, España: McGraw-Hill (p. 11).

Ibarra, A. (2000). Formación de los recursos humanos y competencia laboral. Boletín CINTERFOR, (149) (pp. 96-97). Recuperado 23/06/2008 de: http://www.oei.es/etp/formacion_recursos_humanos_competencia_laboral_ibarra.pdf

Kotler, P., Cámara, D., Grande, I. y Cruz, I. (2000). Dirección de Marketing. Edición Milenio. (1 $1^{\text {ra }}$ reimpresión de la $1^{\text {ra }}$ edición; Moreno, Y., Hamad, J. y Belló, C. Trads.). Madrid, España: Pearson Prentice Hall.

Kotler, P., Bowen, J., Makens, J., Rufín, R. y Reina, M. (2004). Marketing para turismo. ( $3^{\text {ra }}$ reimpresión de la $3^{\text {ra }}$ edición; Belló, C., Blázquez, E. y Nieto, R. Trads.). Madrid, España: Pearson Prentice Hall (p. 25).

Lovelock C., D’andrea G., Huete L. y Reynoso J. (2004). Administración de servicios: Estrategias de marketing, operaciones y recursos humanos. ( $1^{\text {ra }}$ reimpresión de la $4^{\text {ta }}$ edición). Sánchez, M. y Mascaró, P. Trads.). México, D.F., México: Pearson Prentice Hall.

McClelland, D. (1973). Testing for competente rather than for intelligence. American Psychologist, 28 (1) (pp. 1-14).

Mendenhal, W. (1990) Estadística para administradores. $\left(^{\text {ra }}\right.$ reimpresión de la $2^{\text {da }}$ edición; Valckx, D. Trad.). México, D.F., México: Iberoamérica. 
Organización Internacional del Trabajo (OIT). (2005). Recomendación 195 Recomendación sobre el desarrollo de los recursos humanos: educación, formación y aprendizaje permanente. Recuperado 22/03/2007 de: http://www.oitcinterfor.org/ public/spanish/region/ampro/cinterfor/temas/ dialogo/inf_act/rec195.pdf

Organización Mundial del Turismo (OMT) (1999). Código Ético Mundial Para el Turismo. Recuperado 21/04/2007 de: http://www.unwto.org/code ethics/pdf/languages/Codigo\%20Etico\%20Espl. pdf

Padrón, J. (2006). Bases del concepto de "Investigación Aplicada" (o "Investigación Aplicativa" o “Aplicaciones"). Recuperado 01/03/2007 de: http://padron.entretemas.com/InvAplicada/ index.htm

Sáez, S. (2008). Trato especial y diferenciado y comercio de servicios. CEPAL - Serie Comercio internacional, (90) (pp. 28-35). Recuperado 01/03/2009 de: http://www.eclac.org/comercio/publicaciones/ $\mathrm{xml} / 1 / 35301 / \mathrm{serie}$ 90_TRATO_ESPACIAL_DIFERENCIADO_COMERCIO_SERVICIOS.pdf

Zeithaml, V. y Bitner, M. (2002). Marketing de Servicios: Un enfoque de integración del cliente a la empresa. ( $2^{\mathrm{a}}$ reimpresión de la $2^{\mathrm{a}}$ edición; Pérez, M., Lozano, A. y Pontón, S. Trads.). México, D.F., México: McGraw-Hill. 